\title{
Sparkassen und Kreditgenossenschaften - zwei ungleiche Zwillinge?!
}

\section{Ein morphologischer Beitrag zur widmungswirtschaftlichen Problematik öffentlicher oder gemeinwirtschaftlicher Unternehmen}

\section{Vorbemerkungen}

Der Titel drückt das Analyseprogramm aus. Es geht objekttheoretisch gesehen um die These eines Zwillingsstatus von Sparkassen einerseits und Kreditgenossenschaften andererseits. Doch sollen diese ungleich sein. Da der Ungleichheitscharakter sich nicht a priori normativ auf eine Wertigkeitshierarchie bezieht, ist phänotypologisch eher die Unähnlichkeit gemeint. Es geht methodologisch um eine moderne institutionentheoretische Frage, die wir aber in der Art des Fragens rückbinden zu einer für die gängige Wirtschaftslehre unüblichen Forschungstradition.

In der Tradition der Analyse der Logik symbolischer Formen bei Ernst Cassirer (1871-1926) fragen wir nach den Formen (dazu auch Kreis 2009), in denen sich (hier folgen wir dem durch die Wissenssoziologie von Karl Mannheim [1893-1947: dazu auch Barboza 2009] und der Kulturtheorie von Aby Warburg [1866-1929: dazu auch Rösch 2010] geprägten Kunsthistoriker Ernst Panofsky [1892-1968] - vgl. unmittelbar Panofsky 2006) menschliche Zwecksetzungen als Sinngebilde zum Ausdruck bringen. Dabei können die Formen einerseits daseinstechnischen Charakter haben, aber andererseits auch eine tiefere Daseinsqualität anvisieren, wobei das personale Sein des Menschen eine gestaltförmige Wahrheit erhält, der Mensch sich also wesensmäßig entfalten kann.

Unsere eigene Wertsetzung, die hier erkenntnisleitend im Sinne der neu-kantianischen Wissenschaftslehre von Max Weber (1864-1920) ist, aber ihre Wurzeln in fundamentalontologischen Überlegungen hat, die hier nicht zu entfalten sind, knüpft an die Dialogizität des Menschen im sozialen Miteinander an, die vom mutualen Prinzip auf der Grundlage des Gabedispositivs (Schulz-Nieswandt 2010, S. 564 ff.) geprägt ist.

\section{Phänotyp und Genotyp}

Diese phänotypologische Unähnlichkeitsprämisse verknüpft sich nun aber mit der offensichtlichen genotypologischen Prämisse, die beiden wirtschaftlichen Gebilde seien Zwillinge, also in ihrem impliziten Programmcode verwandt, weisen also Gemeinsamkeiten auf. 


\section{Zur Hermeneutik der Morphologie der Gebilde}

Fasst man die Kategorie des Programmcodes im methodologischen Schnittbereich von Systemtheorie und Konstruktivismus, so muss ein verhaltensgenerierender Programmcode als normative Handlungslogik gemeint sein, der vor allem die Identität des eigenen Systems von der Umwelt abgrenzend zu konstituieren hat (kritisch dazu Jung 2009; Farzin 2006).

Greifen wir statt auf diese abstrakte Sprache der Systemtheorie lieber auf die abstrakte Sprache einer hermeneutischen Morphologie zurück (Schulz-Nieswandt 2007 a). Diese altmodisch wirkende theoriegeleitete Terminologie hat den Vorteil, gerade durch die distinktive Wirkung dieser Sprache die nachhaltige Modernität der Gebilde transportieren zu können.

Es geht um eine Gestaltanalyse. Gestalt ist die Ausdruckspraxis, die nur im Rückgriff auf die Hermeneutik (Verstehenslehre und -praxis) des Sinns (Stekeler-Weithofer 2011; Bongaerts 2012) der Gebilde zu analysieren ist. Denn Gestalt ist Ausdrucksform des intentionalen Sinns, der in der Gestalt seine Daseinsqualität verwirklicht. Der Sinn ist die identitätsstiftende Aufgabe, der sich das Gebilde stellt, ihr existenzieller Zweck, auf den hin überhaupt die Notwendigkeit einer Formfindung zwingend ist.

Wichtig ist es zu begreifen, dass die Gestaltwerdung an der Formfindung generativ gebunden ist: Die Form generiert die Daseinsqualität, die mit der Gestaltwerdung der Ausdruckspraxis eines Sinns verbunden ist.

Aus diesen sehr abstrakten Überlegungen aus der Theorie der Daseinshermeneutik kann bereits ein relevanter Schluss gezogen werden:

Der Inhalt ist an eine passende Form gebunden, grenzenloser Form-Opportunismus ist nicht möglich, wohl aber eine gewisse Äqui-Funktionalität verschiedener Formen. Konkretisiert auf die Fragestellung: Sparkassen und Kreditgenossenschaften weisen, strukturmorphologisch gesehen, unterschiedliche Formeigenschaften auf, haben aber, sinnmorphologisch gesehen, Konvergenzen im normativen Programmcode, da es um ähnliche Funktionalitäten geht.

\section{Die Strukturmorphologie im Lichte der älteren rechtsgeschichtlichen Formenlehre}

Auf die Rechtsgeschichte von Otto von Gierke (1841-1921) ${ }^{1}$ geht die Unterscheidung von Herrschaft und Genossenschaft zurück (vgl. in Gierke 1954). Dabei wird eine historische Dynamik der konkreten Figurationen des Mit-, Gegen- oder auch Nebeneinanders konstatiert. Auch (vgl. Kruck 1997) Franz Oppenheimer (1864-1943) im Rückgriff auf die Soziologie der Herrschaft (Breuer 2011) bei Max Weber (1864-1920) wäre zu nennen.

1 Dazu auch Peters 2001. Vor allem aber auch in Schulz-Nieswandt 2003. 


\section{Genossenschaftlichkeit}

Die Genossenschaften sind, soziologisch (und, angesichts der kulturgeschichtlichen wie auch kulturvergleichenden Ubiquität, auch anthropologisch gesehen) unabhängig von einer spezifischen Rechtsform soziale Gebilde der Selbstorganisation zum Zwecke der selbstverwalteten Deckung des Bedarfs der Mitgliederinnen und Mitglieder auf der Basis des Prinzips der Gegenseitigkeit (Reziprozität als Mechanismus von Gabe und Gegen-Gabe; Schulz-Nieswandt $2010 \mathrm{a}$, S. 565 ff.; Schulz-Nieswandt/Alich/Köstler/Mann/Sauer 2009).

Eine asymmetrische Form ist in diesem Austauschprinzip nur eine mögliche Form. Es kann einen dauerhaften Gabe-Überschuss von Teilen der Mitgliedschaft geben und umgekehrt auch NettoNehmer-Positionen. Dies ist wichtig für das Verständnis von Solidarvorgängen innerhalb von Gegenseitigkeitsbeziehungen. Die Austausch“währungen“ können auch homomorph oder heteromorph sein.

Diese morphologische Bestimmung der Genossenschaftlichkeit verweist auf die mitglieder-orientierte Fördergemeinschaftlichkeit des Gebildes. Im Typus der gemeinwirtschaftlich orientierten Widmungsgenossenschaft wird aber auch deutlich, dass die Genossenschaftlichkeit nicht ausschließlich im Rahmen einer Theorie des geschlossenen Interessensclubs zu verstehen ist (dazu auch Schulz-Nieswandt 2011; Schulz-Nieswandt/Alich/Blome-Drees/Köstler/Schmale 2010), sondern positive soziale Externalitäten intentional und direktional zum Sinn des sozialen Gebildes gehören können. An Beispielen wie der Gesundheitsselbsthilfegruppen (Schulz-Nieswandt 2011 a, 2012 c) oder auch der Seniorengenossenschaften (vgl. auch in Schulz-Nieswandt/Köstler 2011) hat das die neuere Forschung aufzeigen können.

Dieser Befund ist wichtig, da so später die (,Als ob“2_-)Instrumentalfunktion (Boos/Krönes 1990) von Genossenschaften (analog zur Sparkasse) gewährleistungsstaatstheoretisch begründet werden kann, ohne hier den Anschluss an moderne Theorien mehrschichtiger Prinzipal-AgentenTheorien suchen zu müssen.

\section{Herrschaft}

Asymmetrien in der sozialen Geometrie der horizontalen Gegenseitigkeit können auch eine Achsendrehung zum Hierarchischen aufweisen, womit Phänomene der Dominanz, der Machtausübung, gar der Herrschaftsbildung wirksam werden können.

Herrschaft ist politische Machtausübung eines dazu organisierten Akteurs, der auf der Basis seines Monopols auf legitime physische Gewalt (kritisch dazu auch Loick 2012) eine Chance auf Huldigung/Zustimmung aufweist. Diese Eigenschaften kommen dem Rechtsstaat (finanzsoziologisch als „Steuerstaat" verstanden) zu, auch in seiner materialen Formbestimmtheit als Sozialstaat.

Konstitutiv für das öffentliche Wirtschaften, auch im Bereich der öffentlich-rechtlichen Körperschaften (historisch: Bieback 1976), sind Steuerungsprinzipien wie die der Dienstherrenfähigkeit, der Satzungs- bzw. Rechtsetzungshoheit oder der Abgabenhoheit (Steuern, Beiträge, Gebühren).

2 Damit spielen wir an auf die neu-kantianisch orientierten Arbeiten von Hans Vaihinger (1852-1933), der („Die Philosophie des Als Ob“, zuerst 1911) zugleich als partieller Vorläufer des Pragmatismus gelten kann, auch einige konstruktivistische Perspektiven bereits bahnend. Vgl. dazu Ceynowa 1993. 
Nicht zuletzt europarechtlich entstehen Probleme (vgl. dazu Schulz-Nieswandt 2010; 2011 b; $2011 \mathrm{c}$ ) zunehmend in Bezug auf hybride Gebilde der Erledigung öffentlicher Aufgaben, bei denen sich die institutionelle (trägerbezogene und rechtsformfundierte) Sichtweise in der Praxis mit funktionellen Sichtweisen konkret vermischt haben. Dies betrifft die vielfältigen Formen der dann zunehmend ,staatsfernen“ Beteiligung Privater an der somit gemischtwirtschaftlichen Leistungserstellung und Aufgabenerledigung (zu den DA[W]I oder DAI vgl. auch Schulz-Nieswandt $2010 \mathrm{c})$.

\section{3. „Genossenschaftliche Herrschaft"}

Die Gierke'sche rechtsgeschichtliche Tradition kennt auch die Tradition der „genossenschaftlichen Herrschaft“. Hier bedient sich der Staat der Genossenschaft als Organisationsprinzip zur Erfüllung delegierter öffentlicher bzw. öffentlich relevanter Aufgaben. In der deutschrechtlichen Tradition ist das eine etablierte Art der Staatlichkeit (Hsu 2004), die den modischeren Neu-Begriff des Gewährleistungsstaates vorwegnimmt (Schulz-Nieswandt/Mann 2009; Schulz-Nieswandt 2008 b). Öffentliche Aufgaben (Mühlenkamp/Schulz-Nieswandt 2008) werden an private oder freie (frei-gemeinwirtschaftliche, eventuell steuerfreigemeinnützige) Träger (die daher in einem regulierten Wettbewerb zueinander/miteinander [Quasi-Markt-Theorem] stehen können) zur Erfüllung/Erledigung delegiert (europarechtlich „,betraut“, eventuell auch in Form von Ausschreibungswettbewerb vergeben) (Schulz-Nieswandt 2010 d).

\section{Explizite und implizite Instrumentalfunktion}

Diese Aufgabendelegation an Verbände und/oder Leistungseinrichtungsträger (Sozialunternehmen) kann explizit erfolgen, aber auch implizit, im gewissen Sinne als fiktionale Szenariumsfigur. Dies trifft für einen großen Teil auch des sog. „Dritten Sektors“ zu (Schulz-Nieswandt 2008); das dort organisierte ehrenamtliche Engamentspektrum wird durch eine „Engagementpolitik“ von Bund, Ländern, Kommunen, zum Teil Stiftungen gefördert. Genossenschaftsartige Selbsthilfegruppen werden auch im Sozialrecht, z. B. im Rahmen des SGB V, auch des SGB XI gefördert. In diesem Sinne wird die neu-kantianische „Als-ob“-Formel genutzt: Wenn die nicht-staatlichen Akteure die Leistungserstellung im Rahmen vielfältiger PPP-Settings nicht übernehmen und erledigen würden (oder darin scheitern), müsste, falls die öffentliche Relevanz der Güter bzw. Dienstleistungen (Mühlenkamp/Schulz-Nieswandt 2008) entsprechend politisch wertgeschätzt wird, diese vom Staat in eigener Regie übernommen werden. Beispiele finden sich in der neueren Re-Kommunalisierungs-Praxis. Insofern kommen die nicht-staatlichen Träger (etwa auch große Teile des sog. „Dritten Sektors“) in die Rolle von staatsmittelbaren Akteuren. Es handelt sich um nicht-materielle, aber organisationsformale Privatisierungen, besser: „Vergesellschaftungen“, da es sich nicht a priori um die Delegation an private erwerbswirtschaftliche Marktteilnehmer handeln muss. 


\section{Das instrumentalfunktionslogische „Geschäftsmodell“ der Sparkassen und der Genossenschaften}

Die Besonderung der Sparkassen im Feld der Konkurrenzsituation zu allen anderen Akteuren von Angeboten, Produkten und Geschäftsfeldern der Bankenlandschaft ist historisch zu verstehen (Braun 2011) und resultiert aus der besonderen Gemeinwohlbindung, die Sparkassen nicht als „nur wirtschaftliche“ (d. h. erwerbswirtschaftliche) Unternehmen erscheinen lassen. Regionale Wirtschaftsförderung, die Förderung kleiner und mittlerer Betriebe (Leiß1 2011), aber auch soziales Engagement und gemeinnützige Projekte gehören zum Identitätsprofil. Hier liegt ihr öffentlicher Auftrag begründet (Wissenschaftsförderung der Sparkassen-Finanzgruppe 2010).

Die Instrumentalfunktion der deutschen Sparkassen ist mit Blick auch auf normativ-empirische Differenziale (also im Sinne einer Soll-Ist-Vergleichsanalyse) nochmals gründlich dargelegt worden bei Brämer/Gischer/Pfingsten/Richter 2010 sowie in der 2007er Dissertation von Handschuh (2010). Dabei werden auch marktversagenstheoretische Argumente herangezogen und institutionenökonomische Aspekte beachtet.

Dennoch wird kritische Wissenschaft, auch dann, wenn sie sich in ihren transzendentalen Wertbezügen der Sparkassenidee, allgemein: der notwendigen Idee der Erledigung öffentlicher Aufgaben, widmet, die Norm nicht für die soziale Realität halten. Die empirischen Differenziale (zwischen Anspruch und Wirklichkeit: Konrads 2007) evozieren natürlich Privatisierungsdiskurse und -praktiken (Schorner 2008; Dietl 2010). Und angesichts der anstehenden Anpassungsprobleme der nahen Zukunft (etwa die Angebotspräsenzpolitik angesichts des sozio-demographischen Wandels, der auch Abwanderung, Schrumpfungsprozesse und teilräumliche AlterungsEntleerungs-Prozesse umfasst: Conrad 2010) ${ }^{3}$ werden Bewährungsproben wirksam. Hier ist die Rolle von Aufgaben vom Typ der sozialen Dienste auch zu einem Handlungsfeld öffentlicher oder gemeinwirtschaftlicher Unternehmen im Bereich der Gewährleistung monetärer Infrastrukturen geworden.

\section{Idealtypus oder Realtypus}

Der Befund normativ-empirischer Differenziale verweist darauf, dass die Idee der Sparkasse realtypologisch zu verstehen ist. Nicht gemeint und wirksam ist hier die Max Weber'sche (1864-1920) Methode des Idealtypus (Gerhardt 2001), die seine Wissenschaftslehre im Wirkkontext des südwestdeutschen Neu-Kantianismus (Merz-Benz 1990) entwickelt hat. Beim Idealtypus werden absichtlich bestimmte Aspekte realer Phänome in extremer Stilisierung übersteigert. Es geht daher in der Forschung nicht um eine empirische Abbildungsqualität der sozialen Realität, sondern um einen Typusgewinn, der im Lichte transzendentaler Wertsetzungen von hoher Kulturbedeutsamkeit leitend sein soll.

3 Ähnliche Probleme stellen sich auch in anderen Bereichen der sozialen Infrastruktur-Gewährleistung, z. B. im Bereich der ambulanten medizinischen Versorgung in (bestimmten) ländlichen Räumen. Auch in der Vorhaltung allgemeinbildender Schulen ist dieses Phänomen schon länger beobachtbar. Vgl. etwa auch Süßmuth 2012. 
Ein Realtypus abstrahiert von vielerlei Facetten des realen Phänomens, behält jedoch die erkenntnislogische Absicht bei, wesentliche soziale Realität ,richtig“ abzubilden.

\section{Morphologie der Sparkasse}

Sparkassen (im Rahmen des deutschen Systems der Drei-Säulen-Architektur des Finanzsektors) als Kreditinstitute haben kreditwirtschaftliche Aufgaben, wobei die Gewinnerzielung nicht der Hauptzweck darstellt. Das Geschäftsfeld ist in der Regel regional orientiert, somit auf die Örtlichkeit des Trägers, der Gemeinde, des Landkreises oder eines inter-kommunalen Zweckverbandes ${ }^{4}$ begrenzt. ${ }^{5}$ Hier zeichnet sich instrumentalfunktionslogisch der Zusammenhang mit der Idee der kommunalen Daseinsvorsorge ab. Historisch kommen die Motive der Förderung von Kleinsparern konstitutiv hinzu.

Die Bedeutung nicht nur flächendeckender Einrichtungsvorhaltung, sondern auch die Bedeutung der Sicherstellung der Zugangschancen zu einem Girokonto (Niekiel 2011) wird in der einschlägigen Literatur im Zusammenhang mit (über-)schuldeten privaten Haushalten betont, womit ein weiteres sozial- und gesellschaftspolitisch brisantes Thema angesprochen ist (dazu auch SchulzNieswandt/Kurscheid 2007). ${ }^{6}$

Rechtlich sind Sparkassen in der Regel (Quast 2010; Bowmann 2010; Stolzmann 2010; Scheike 2004) als öffentlich-rechtliche Institute (als Anstalten des öffentlichen Rechts) organisiert. Leitmotiv ist die sachziel-dominante Gemeinwohlbindung und-förderung. Gewinne finden ihre Verwendung im Rahmen von Sicherheitsrücklagen, trägerorientierten Ausschüttungen (Lepper 2003) oder Stiftungen bzw. anderen sozialen Engagementformen.

Das Sicherungssystem der Sparkassen-Finanzgruppe bleibt hier außerhalb der Betrachtung; auch auf die gewandelte Situation und Rolle der Landesbanken wird hier nicht weiter eingegangen (Staats 2006).

Die Instrumentalfunktion ist im Fall der Sparklasse evident, denn die Anstalt des öffentlichen Rechts (die zum Teil auch für kommunale Krankenhäuser als Rechtsform genutzt wird: Rinken 2008), wird mit öffentlichen Aufgaben (auch bundes- oder landesmittelbar) betraut (definiert als Hoheitsakt). Gewährleistung des Trägers bedeutet hier Anstaltslastverpflichtung, was europarechtlich als (wettbewerbs-, vergabe- und beihilferechtlich) problematisch ausgelegt wird (Kemmler 2001; Sommerfeld 2005; Weinkamm 2004; Kinzl 2000).

4 Dazu auch Loer 2007; Schmidt 2005; Bräutigam 2009.

5 Dieses Örtlichkeitsprinzip vertreten wir auch mit Blick auf das kommunale Wirtschaften im Lichte des EU-Rechts. Dazu die breite Diskussion etwa bei Scheps 2006; Langner 2008; Wenzl 2007; Rode 2006.

6 Zur Problematik der Schuldnerberatung vgl. ferner Thomsen 2008; Gastiger/Stark 2011; Schruth u. a. 2011; Ebli 2003; Schlabs 2006. 


\section{Morphologie des Genossenschaftlichen}

Die Genossenschaften, von denen hier die Rede ist, meinen soziologisch (nicht rechtlich) eine Gebildeart, die durchaus eine Vielzahl des Gestalt-Werdens der Gabe und der Gegenseitigkeit als moralökonomische Modi der Daseins-Werdung der Person zum Ausdruck bringt.

Einerseits verstehen wir, wir folgen hier der Draheim'schen Begrifflichkeit (Draheim 1955), Genossenschaften als wirtschaftliche Betriebsgebilde, andererseits sind sie Personenverbände, (womit Georg Draheim [1903-1972] eine Anlehnung an Max Weber [1864-1920] vornimmt), als Verbände von Personen zur Durchführung von Gegenseitigkeitshilfen, die eine Wirtschaftsart eigener Art darstellen. Über das personenverbandliche Wesen der Assoziationen hat der bereits angeführte Otto von Gierke ebenso grundlegend nachgedacht wie spätere Beiträge etwa von Georg Weippert. Diese daseinsontologische und anthropologische Sichtweise, die von einem ,methodologischen Personalismus“ geleitet ist, soll hier nicht weiter ausgebreitet werden. Sie wird Gegenstand einer umfassenden Abhandlung „Gemeinwirtschaft, Genossenschaft, Selbsthilfe, Person. Hermeneutik und Morphologie“ (Berlin: Duncker \& Humblot, i. V.) sein. Wichtige Grundgedanken sind bereits artikuliert (Schulz-Nieswandt 2012; 2012 a). An dieser Stelle soll auch nicht umfassend auf die genossenschaftswissenschaftliche Literatur verwiesen werden (vgl. Brockmeier/Fehl 2007).

Der Personalbezug der Genossenschaftsidee wird heute zum Teil im Kontext einer werte-orientierten wirtschaftlichen Betätigung abgehandelt (Eichwald/Lutz 2011, Janßen 2011). Hierbei geht es um kulturelle Einbettungen (Daheim/Kruse 2008), die sich auf die lokale Einbindung der Kreditgenossenschaften bezieht, mit der wiederum Aspekte der Nähe, des Vertrauens, der emotionalen Bindung sowie Verankerungen im lokalen Vereinswesen etc. verbunden sind.

Die Kreditgenossenschaften in Deutschland in der Rechtsform der eingetragenen Genossenschaft mit ihren Grundsätzen der Selbsthilfe, der Gegenseitigkeitssolidarität und der Selbstverwaltung wirken heute im Rahmen ihrer flächendeckenden Struktur, in der ihr intentionaler Sozialraumbezug (Kessl u. a. 2005) zum Ausdruck kommt. Auf die Ebene des Finanzverbundes soll hier nicht weiter eingegangen werden.

\section{Wirtschaftstehische Dynamiken}

Corporate Social Responsibility-Aktivitäten im Kontext von Corporate Citizenship-Denk- und Praxisansätzen (Backhaus-Maul/Biedermann/Nährlich/Polterauer 2010; Braun/Backhaus-Maul 2010) sind daher sowohl bei den Kreditgenossenschaften (Roth 2006) wie auch bei den Sparkassen (ebenso auch bei kommunalen Unternehmen: Sandberg/Lederer 2011) ausgeprägte Themen.

Man kann insgesamt von einer SCR-Bewegung sprechen (Curbach 2009). Und insbesondere mit Blick auf die Finanzmärkte haben diese Themen in großer Dichte zugenommen.

Nur kurz sein angemerkt: Methodologisch haben somit verschiedene paradigmatische Varianten der Wirtschafts- und Unternehmensethik (Aßländer 2011) an Relevanz gewonnen, indem nicht nur anreizökonomische Ansätze, sondern auch (Schulz-Nieswandt 2008 a) eher kulturanthropologisch orientierte Ansätze der kulturellen Einbettung norm- und werteorientierten sozialen Handelns sowie der Denkstile und der orientierenden Deutungsmuster an Bedeutung gewonnen haben. 


\section{Das Formprinzip des Genossenschaftlichen}

Die Sparkasse ist Instrument staatlicher Herrschaft. Ihre Rechtsform ermöglicht manageriale Freiheitsgrade im konkreten Wirtschaften. Sinnbezogen bleibt die Sparkasse aber an den öffentlichen Auftrag gebunden. Das verhindert im historischen Wandel nicht unbedingt Transformationsprozesse im Sinne der Konvergenz zum erwerbswirtschaftlichen Verhaltensmuster. In der Regel liegen hier Habitus-Transformationen des Managements vor, die durch defizitäre Dienstgesinnung (Schulz-Nieswandt 2008 a) bedingt sind und durch sinnfremde normative Orientierungen am Referenzmodell der Privatwirtschaft erklärbar sind (Katterle 1988). Das Transformationsgesetz von Franz Oppenheimer (1864-1943) hat diese Prozesse an Produktivgenossenschaften, eingebettet aber auch in „Siedlungsgenossenschaften“, beobachtet (Kruck 1997).

Die soziale Tatsache der Instrumentalfunktionslogik eröffnet institutionelle Freiheitsgrade in der Ausgestaltung der Wahrnehmung der öffentlichen Aufgabe. In diesem Lichte eröffnet sich im Kontext vor allem der europäischen Rechtsregimeentwicklung die Problematik einer engen Inhouse-Auslegungsordnung (Schulz-Nieswandt 2012 b), so dass sich der hegemoniale Mainstream-Diskurs in Richtung auf eine gewährleistungsstaatliche Interpretation öffentlicher Aufgaben hinbewegt, deren Erfüllung (Aufgabenerledigung) aber marktorientiert wettbewerblich erfolgen soll. Auf die Details soll hier nicht weiter eingegangen werden (Schulz-Nieswandt 2011 b; 2012 d; 2010 b). Die Form der Aufgabenerledigung wird damit flexibel und zur Frage relativer Kosten-Effektivität in unterschiedlichen transaktionskostenökonomischen Settings.

Im Fall des Genossenschaftlichen liegt die Formproblematik anders. Dazu muss nochmals auf die daseinsontologischen Überlegungen zum Form-Inhalts-Problem (Schulz-Nieswandt 2007) zurückgekommen werden.

Nicht jeder Inhalt kann sich in beliebig vielen unterschiedlichen Formen daseinsadäquat zum Ausdruck bringen. Zwischen Sparkassen und Kreditgenossenschaften haben wir eine gewisse Äqui-Funktionalität konstatiert, wenn es um die Instrumentalfunktionen (Regionalpolitik, Strukturpolitik, personelle Nähe im örtlichen Sozialraumbezug etc.) geht. Aber im Dasein des Genossenschaftlichen hat die Form eine andere Bedeutung. Die Form selbst ist nicht nur Form eines Inhalts, der zum Ausdruck kommt. Dies gilt in fundamentaler Art auch: Das soziale Mit-Sein ermöglicht das personale Dasein. Personalität kommt in der Praxis der solidarischen Gegenseitigkeit zur sozialen Wirklichkeit. Aber die Form generiert dies personale Sein als Praxisort der Gestaltqualität der Person. Diese Generativität sichert der Form des Genossenschaftlichen einen Eigenwert, der über die Funktionalität einer Form für den (eigentlichen) Inhalt hinausgeht und traditionelle zwei-stellige hyhlemorphe Denkmodelle überwindet.

Das Telos des Person-Seins im Modus des sozialen Mit-Sein ist in dieser Form-Inhalts-Metaphysik zwar die Substanz, die sich in der Form aktualisiert. Doch generiert die Form überhaupt erst (performativ) die Formwerdung des Inhalts. Der Form kommt somit Wahrheitscharakter als Gestaltqualität des Daseins zu. 


\section{Zusammenfassung}

Stellen wir realtypologisch Genossenschaft und die öffentlich-rechtliche Körperschaft gegenüber, so werden Wahlverwandtschaften deutlich.

Entstehung: Ein erster Unterschied besteht in der Logik der Soziogenese: ${ }^{7}$ Die Genossenschaft resultiert aus einem assoziativen Ursprungsprozess, die Körperschaft des öffentlichen Rechts folgt aus einem hoheitlichen Akt.

Funktionalität: In instrumentalfunktionstheoretischer Perspektive besteht eine Differenz innerhalb einer Analogik: Die Genossenschaft ist ein „offener Club“ mit gemeinwirtschaftlichen Externalitäten, da a) die Form der internen Moralökonomik öffentlich wertzuschätzen ist und b) widmungswirtschaftlich innerhalb dieser Fördergemeinschaftlichkeit öffentlich relevante Bedarfe gedeckt werden, wodurch eine „Als ob“-Instrumentalfunktion vorliegt: Es werden öffentlich relevante Aufgaben erfüllt, die, wenn nicht so, dann genuin öffentlich erfüllt werden müssten. Die Körperschaft des öffentlichen Rechts hat explizit eine öffentliche Aufgabe in dieser ihr eigenen Form zu erfüllen.

„Konvergenztrieb“: Beiden Formen gemeinsam ist ein Norm-Empirie-Differential im Sinne eines Soll-Ist-Vergleichs und damit ein permanentes Transformationspotenzial, das hier als „Konvergenztrieb" bezeichnet werden soll.

Haltungspädagogische Basis: Daraus resultiert als gesellschaftspädagogische Voraussetzung des jeweiligen Organisationsgelingens in der Genossenschaft die notwendige Haltung des homo kooperativus, in der Körperschaft des öffentlichen Rechts die Haltung der Dienstgesinnung. Die Haltungen sind (mutualistische Haltung versus asymmetrische Haltung) unterschiedlich (Modus des gegenseitigen Helfens versus Verpflichtung auf das Dienen, dem Träger und in diesem Lichte „Dritten“). Die Mutualität ist in Bezug auf die Insider-Gemeinschaft unmittelbar und nur mittelbar Dritten gewidmet; der Dienst ist dagegen unmittelbar dem Auftrag und daher dem Träger gewidmet und erst mittelbar den Dritten.

Stiftung sozialen Nutzens durch soziales Engagement: Beide Formen neigen aus formgebundener Kultur der Gemeinwohl-Wertschätzung heraus zum sozialen Engagement.

In der Form der Genossenschaft ist das gelebte Reziprozitätsprinzip selbst bereits ein Praxisort des sozialen Lernens, in der Form der Körperschaft des öffentlichen Rechts ist soziales Engagement als Modus der Gewinnverwendung ein gewollter Stakeholder-orientierter OrganisationsHabitus.

Auch die Genossenschaft kann aus ihrer formgebundenen Kultur der Gemeinwohl-Wertschätzung heraus für „Dritte“ soziales Engagement zeigen. Anzufügen wäre hier auch die nach neustem deutschen Genossenschaftsrecht mögliche Wahl der eingeschriebenen Genossenschaft als Rechtsform möglicher Betätigung im sozialen und kulturellen Handlungsfeld.

Formprinzip: Und geradezu in transzendentaler Art ist das jeweilige Formprinzip zu verstehen: Das Genossenschaftliche ist expressiv und generativ zugleich: Es dient der institutionellen Verwirklichung als Ausdruckspraxis des „Gewollten“ (Exklusivitätscharakter), ist aber zugleich generativ: Die Form drückt einen gewollten Inhalt nicht nur (passiv) aus, sondern ist in ihrer Präsenz

7 Zur realen Sozialgeschichte vgl. auch Wysocki 2005; Pohl/Rudolph/Schulz 2005. 
ein performativer Akt der Erzeugung des „Gewollten“. Die Körperschaft als Form ist Instrument des Gewollten und insofern „dienender“ Art, keine eigenständige Formwertigkeit. Die Körperschaft ist eine inhaltsdominierte Form, die Genossenschaft eine eigenwertige Form, die die Formwerdung eines Inhalts überhaupt erst ermöglicht und über diesen Instrumentalcharakter sittlich zugleich in sich selbst ruht.

\section{Literaturverzeichnis}

Aßländer, M. S. (Hrsg.) (2011), Handbuch Wirtschaftsethik. Stuttgart-Weimar: Metzler.

Backhaus-Maul, H./Biedermann, Chr./Nährlich, St./Polterauer, J. (Hrsg.) (2010), Corporate Citizenship in Deutschland. 2., akt. u. erw. Aufl. Wiesbaden: VS.

Barboza, A. (2009), Karl Mannheim. Konstanz: UVK.

Bieback, K.-J. (1976), Die öffentliche Körperschaft. Ihre Entstehung, die Entwicklung ihres Begriffs und die Lehre vom Staat und den innerstaatlichen Verbänden in der Epoche des Konstitutionalismus in Deutschland. Berlin: Duncker \& Humblot.

Bongaerts, G. (2012), Sinn. Bielefeld: transcript.

Boos, F. X./Krönes, G. (1990), Die Instrumentalfunktion öffentlicher Unternehmen: Hauptprobleme und Lösungsansätze, in: Zeitschrift für öffentliche und gemeinwirtschaftliche Unternehmen 13 (2), S. 141-157.

Bowmann, A. (2010), Sparkassenprivatisierungsverbot durch den Landessparkassengesetzgeber. Hamburg: Kovac.

Brämer, P./Gischer, H./Pfingsten, A./Richter, T. (2010), Der öffentliche Auftrag der deutschen Sparkassen aus der Perspektive des Stakeholder-Managements, in: Zeitschrift für öffentliche und gemeinwirtschaftliche Unternehmen 33 (4), S. 311-332.

Bräutigam, F. (2009), Der „Grenzüberschreitende örtliche Zweckverband“ nach dem Karlsruher Übereinkommen. Baden-Baden: Nomos.

Braun, M. (2011), Die Validierung der Sparkassenidee. Eine sozialgeschichtliche Betrachtung. Marburg: Tectum.

Braun, S./Backhaus-Maul, H. (2010), Gesellschaftliches Engagement von Unternehmen in Deutschland. Eine sozialwissenschaftliche Sekundäranalyse. Wiesbaden: VS.

Breuer, St. (2011), „Herrschaft“ in der Soziologie Max Webers. Wiesbaden: Harrassowitz.

Brockmeier, Th./Fehl, U. (Hrsg.) (2007), Volkswirtschaftliche Theorie der Kooperation in Genossenschaften. Göttingen: Vandenhoeck \& Ruprecht.

Ceynowa, K. (1993), Zwischen Pragmatismus und Fiktionalismus. Hans Vaihingers „Philosophie des Als OB“. Würzburg: Königshausen \& Neumann.

Conrad, A. (2010), Banking in schrumpfenden Regionen. Hamburg: Kovac.

Curbach, J. (2009), Die Corporate-Social-Responsibility-Bewegung. Wiesbaden: VS.

Daheim, H./Kruse, V. (2008), Kreditgenossenschaften in Deutschland. Hamburg: Kovac.

Dietl, Chr. J. (2010), Das Ende der Anstaltslast und Gewährträgerhaftung. Duisburg-Hamborn: WiKu.

Draheim, G. (1955), Die Genossenschaft als Unternehmenstyp. 2. Aufl. Göttingen: Vandenhoeck \& Ruprecht.

Ebli, H. (2003), Pädagogisierung, Entpolitisierung und Verwaltung eines gesellschaftlichen Problems? Die Institutionalisierung des Arbeitsfeldes Schuldnerberatung. Baden-Baden: Nomos.

Eichwald, B./Lutz, K. J. (2011), Erfolgsmodell Genossenschaften. Möglichkeiten für eine werteorientierte Marktwirtschaft. Wiesbaden: Deutscher Genossenschafts-Verlag.

Farzin, S. (2006), Inklusion/Exklusion. Entwicklungen und Probleme einer systemtheoretischen Unterscheidung. Bielefeld: transcript.

Gastiger, S./Stark, M. (Hrsg.) (2011), Schuldnerberatung - eine ganzheitliche Aufgabe für methodische Sozialarbeit. Freiburg i. Br.: Lambertus.

Gerhardt, U. (2001), Idealtypus. Zur methodischen Begründung der modernen Soziologie. Frankfurt am Main: Suhrkamp.

Gierke, O. von (1954), Das Genossenschaftsrecht, Bd. 1: Rechtsgeschichte der deutschen Genossenschaft (Berlin 1868). Nachdruck: Darmstadt: WBG.

Handschuh, G. A. (2010), Der öffentliche Auftrag der sächsischen Sparkassen. Baden-Baden: Nomos.

Hsu, C.-C. (2004), Institutionen körperschaftlicher Selbstverwaltung. Hamburg: Kovac.

Janßen, St. (2011), Wert(e)orientierung in Genossenschaftsbanken. Göttingen: Sierke.

Jung, A. (2009), Identität und Differenz. Sinnprobleme der differenzlogischen Systemtheorie. Bielefeld: transcript.

Katterle, S. (1988), Ethische Aspekte des Verhaltens von Führungskräften öffentlicher und gemeinwirtschaftlicher Unternehmen, in: Zeitschrift für öffentliche und gemeinwirtschaftliche Unternehmen 11 (4), S. 434-447.

Kemmler, I. (2001), Die Anstaltslast. Berlin: Duncker \& Humblot.

Kessl, F. u. a. (Hrsg.) (2005), Handbuch Sozialraum. Wiesbaden: VS.

Kinzl, U. P. (2000), Anstaltslast und Gewährträgerhaftung. Baden-Baden: Nomos. 
Sparkassen und Kreditgenossenschaften - zwei ungleiche Zwillinge?!

Konrads, O. (2007), Die Mittelstandsförderung der Sparkassenorganisation - Anspruch und Wirklichkeit. Frankfurt am Main: Lang.

Kreis, G. (2009), Cassirer und die Formen des Geistes. Frankfurt am Main: Suhrkamp.

Kruck, W. (1997), Franz Oppenheimer - Vordenker der Sozialen Marktwirtschaft und Selbsthilfegesellschaft. Berlin: BWV.

Langner, M. V. (2008), Die örtliche Begrenzung kommunaler Wirtschaftstätigkeit und die Grundfreiheiten des EGVertrages. Frankfurt am Main: Lang.

Leiß1, A. (2011), Der Beitrag der Kreditgenossenschaften zur Finanzierung kleiner und mittlerer Unternehmen. Hamburg: Kovac.

Lepper, M. (Hrsg.) (2003), Die Verwendung und insbesondere die Ausschüttung von Sparkassengewinnen. BadenBaden: Nomos.

Loer, E. (2007), Public Private Partnership und Public Public partnership. Kooperations- und Konzessionsmodelle sowie interkommunale Zusammenarbeit im Lichte des Vergaberechts. Göttingen: V\&R unipress.

Loick, D. (2012), Kritik der Souveränität. Frankfurt am main/New York: Campus.

Merz-Benz, P.-U. (1990), Max Weber und Henrich Rickert. Die erkenntniskritischen Grundlagen der verstehenden Soziologie. Würzburg: Königshausen \& Neumann.

Mühlenkamp, H./Schulz-Nieswandt, F. (2008), Öffentlicher Auftrag und Public Corporate Governance, in: Schaefer, Chr./Theuvsen, L. (Hrsg.): Public Corporate Governance: Bestandsaufnahme und Perspektiven. Zeitschrift für öffentliche und gemeinwirtschaftliche Unternehmen, Beiheft 36. Baden-Baden: Nomos, S. 26-44.

Niekiel, O. (201), Das Recht auf ein Girokonto. Baden-Baden: Nomos.

Panofsky, E. (2006), Ikonographie und Ikonologie. Köln: DuMont.

Peters, M. (2001), Die Genossenschaftstheorie Otto v. Gierkes (1841-1921). Göttingen: Vandenhoeck \& Ruprecht.

Pohl, H./Rudolph, B./Schulz, G. (2005), Wirtschafts- und Sozialgeschichte der deutschen Sparkassen im 20. Jahrhundert. Stuttgart: Deutscher Sparkassen Verlag.

Quast, F. A. (2010), Private als Sparkassenträger. Zur Verfassungskonformität des sog. Berliner Sparkassenmodells. Berlin: Duncker \& Humblot.

Rinken, A. (2008), Alternativen zur Privatisierung. Das selbständige Kommunalunternehmen als Organisationsform der kommunalen Daseinsvorsorge am Beispiel der kommunalen Krankenhäuser. Baden-Baden: Nomos.

Rode, A. (2006), Räumlicher Wirkungskreis kommunaler Unternehmen. Aachen: Shaker.

Rösch, P. (2010), Aby Warburg. München: Fink (UTB).

Roth, K. (2006), Corporate Citizenship von Kreditgenossenschaften in Deutschland. Berlin: LIT.

Sandberg, B./Lederer, K. (Hrsg.) (2011), Corporate Social Responsibility in kommunalen Unternehmen. Wiesbaden: VS.

Scheike, A. (2004), Rechtliche Voraussetzungen für die materielle Privatisierung kommunaler Sparkassen. Frankfurt am Main: Lang.

Scheps, C. (2006), Das Örtlichkeitsprinzip im kommunalen Wirtschaftsrecht. Berlin: Duncker \& Humblot.

Schlaps, S. (2006), Schuldnerinnen - eine biographische Untersuchung. Opladen-Farmington Hills: Barbara Budrich.

Schmidt, Th. I. (2005), Kommunale Kooperation. Tübingen: Mohr Siebeck.

Schorner, F. (2008), Privatisierung kommunaler Sparkassen. Aktuelle Entwicklungen im historischen und internationalen Kontext. Hamburg: Kovac.

Schruth, P. u. a. (2011), Schuldnerberatung in der Sozialen Arbeit. Weinheim-München: Juventa.

Schulz-Nieswandt, F. (2003), Herrschaft und Genossenschaft. Berlin: Duncker \& Humblot.

Schulz-Nieswandt, F. (2007), Die Unbedingtheit der Gabeethik und die Profanität der Gegenseitigkeitsökonomik. Die genossenschaftliche Betriebsform als Entfaltungskontext der menschlichen Persönlichkeit im Lichte einer Form-Inhalts-Metaphysik, in: Rösner, H. J./Schulz-Nieswandt, F. (Hrsg.): Zur Relevanz des genossenschaftlichen Selbsthilfegedankens. 80 Jahre Seminar für Genossenschaftswesen der Universität zu Köln. Berlin: LIT, S. 57-92.

Schulz-Nieswandt, F. (2007 a), Zur Relevanz des betriebsmorphologischen Denkens. Versuch einer sozialontologischen und anthropologischen Grundlegung, in: Bräunig, D./Greiling, D. (Hrsg.): Stand und Perspektiven der Öffentlichen Betriebswirtschaftslehre II. FS für Prof. Dr. Dr. h. c. mult. Peter Eichhorn anlässlich seiner Emeritierung. Berlin: BWV, S. 58-67.

Schulz-Nieswandt, F. (2008), Zur Morphologie des Dritten Sektors im Gefüge zwischen Staat, Markt und Familie. Ein Diskussionsbeitrag zur Ciriec-Studie „Die Sozialwirtschaft in der Europäischen Union“, in: Zeitschrift für öffentliche und gemeinwirtschaftliche Unternehmen 31 (3), S. 323-336.

Schulz-Nieswandt, F. (2008 a), Zur Einführung: Ein Corporate Governance Kodex für das öffentliche Wirtschaften?, in: GÖW (Hrsg.): Corporate Governance in der öffentlichen Wirtschaft. Beiträge zur öffentlichen Wirtschaft, Bd. 27. Berlin: GÖW, S. 7-18.

Schulz-Nieswandt, F. (2008 b), Neuere Literatur zum Wandel der Staatlichkeit, dargelegt im Bezugskreis der europarechtlichen Neu-Adjustierung der (insbesondere sozialen) Dienstleistungen von allgemeinem (wirtschaftlichen) Interesse. In: Zeitschrift für öffentliche und gemeinwirtschaftliche Unternehmen 31 (4), S. 438-452.

Schulz-Nieswandt, F. (2010), Öffentliche Daseinsvorsorge und Existentialismus. Eine gouvernementale Analyse unter besonderer Berücksichtigung der Wasserversorgung. Baden-Baden: Nomos.

Schulz-Nieswandt, F. (2010 a), Wandel der Medizinkultur? Berlin: Duncker \& Humblot. 
Schulz-Nieswandt, F. (2010 b), Daseinsvorsorge und existenzielle Angst des Menschen, in: Jens, U./Romahn, H. (Hrsg.): Methodenpluralismus in den Wirtschaftswissenschaften. Marburg: Metropolis, S. 213-45.

Schulz-Nieswandt, F. (2010 c), The dynamics of European definition policy oh health and social services as services of general (economic) interests, in: Zeitschrift für öffentliche und gemeinwirtschaftliche Unternehmen 33 (1), S. 31-43.

Schulz-Nieswandt, F. (2010 d), Zentrale Themenfelder der Entwicklung des öffentlichen (und frei-gemeinwirtschaftlichen) Sektors in der neueren Literatur, in: Zeitschrift für öffentliche und gemeinwirtschaftliche Unternehmen 33 (4), S. 403-414.

Schulz-Nieswandt, F. (2011), Bemerkungen zur gemeinwirtschaftlichen, einschließlich genossenschaftlichen Einzelwirtschaftslehre und Sozialpolitikwissenschaft im System der Wissenschaft von der Gesellschaftsgestaltungspolitik. Werner Wilhelm Engelhardt zum 85. Geburtstag. in: Zeitschrift für öffentliche und gemeinwirtschaftliche Unternehmen 34 (1), S. 100-109.

Schulz-Nieswandt, F. (2011 a), Gesundheitsselbsthilfegruppen und ihre Selbsthilfeorganisationen in Deutschland. Der Stand der Forschung im Lichte der Kölner Wissenschaft von der Sozialpolitik und des Genossenschaftswesens. Baden-Baden: Nomos.

Schulz-Nieswandt, F. (2011 b), „Europäisierung“ der Sozialpolitik und der sozialen Daseinsvorsorge? Eine kultursoziologische Analyse der Genese einer solidarischen Rechtsgenossenschaft. Berlin: Duncker \& Humblot.

Schulz-Nieswandt, F. (2011 c), Berufsgenossenschaften und Europarecht. Eine sozialökonomische Analyse. Berlin: Duncker \& Humblot.

Schulz-Nieswandt, F. (2012), Gemeinschaftliches Wohnen im Alter in der Kommune. Das Problem der kommunalen Gastfreundschaftskultur gegenüber dem homo patiens. Berlin: Duncker \& Humblot.

Schulz-Nieswandt, F. (2012 a), Der leidende Mensch in der Gemeinde als Rechts- und Hilfegenossenschaft. Berlin: Duncker \& Humblot (i. V.).

Schulz-Nieswandt, F. (2012 b), Der Querverbund im Kontext kommunalen Wirtschaftens, in: Bräunig, D./Gottschalck, W. (Hrsg.): Stadtwerke. Grundlagen, Rahmenbedingungen, Führung und Betrieb. Baden-Baden: Nomos, S. 181-198.

Schulz-Nieswandt, F. (2012 c), Multi-disziplinärer Blick auf soziale gegenseitige Selbsthilfe und politische Selbstorganisation von Menschen mit chronischen und seltenen Erkrankungen, in: Monitor Versorgungsforschung 5 (1), S. 38-42.

Schulz-Nieswandt, F. (2012 d), Institutionelle Präferenzen der Bürger hinsichtlich der Erstellung kommunaler Daseinsvorsorgegüter - Eine tiefenpsychologische Re-Interpretation quantitativer Befragungsdaten, in: Schaefer, Chr./Theuvsen, L. (Hrsg.: Renaissance öffentlicher Wirtschaft. Baden-Baden: Nomos, S. 119-142.

Schulz-Nieswandt F./Alich, A./Köstler, U./Mann, K./Sauer, M. (2009), Generationenbeziehungen. Netzwerke zwischen Gabebereitschaft und Gegenseitigkeitsprinzip. Berlin: LIT.

Schulz-Nieswandt, F./Alich, A./Blome-Drees, J./Köstler, U./Schmale, I. (2010), Gemeinwirtschaftliche Genossenschaftlichkeit - Das Beispiel der Gesundheitsselbsthilfegruppen. Unter besonderer Berücksichtigung der Typusbestimmung von Werner Wilhelm Engelhardt, in: Zeitschrift für öffentliche und gemeinwirtschaftliche Unternehmen 33 (2), S. 122-158.

Schulz-Nieswandt, F./Köstler, U. (2011), Bürgerschaftliches Engagement im Alter. Stuttgart: Kohlhammer.

Schulz-Nieswandt, F./Kurscheid, C. (2007), Die Schuld an der Schuld. Hamburg: Merus.

Schulz-Nieswandt F./Mann, K. (2009), Zur Morphologie der Staatlichkeit im Wandel, in: Zeitschrift für öffentliche und gemeinwirtschaftliche Unternehmen 32 (2), S. 183-202.

Sommerfeld, O. (2005), Wettbewerb kontra Daseinsvorsorge. Die Strukturmerkmale der kommunalen Sparkassen in Deutschland im Lichte des EG-Wettbewerbsrechts. Hamburg: Kovac.

Staats, St. (2006), Fusionen bei Sparkassen und Landesbanken. Berlin: Duncker \& Humblot.

Stekeler-Weithofer, P. (2011), Sinn. Berlin-New York: de Gruyter.

Stolzmann, Chr. (2010), Privatisierung von Sparkassen. Die Rolle der Sparkassen im deutschen Bankensystem. Tönning: Der Andere Verlag.

Süßmuth, A. (2012), Sicherstellung der Gesundheitsversorgung im ländlichen Raum. München: Akademische Verlagsgemeinschaft.

Thomsen, M. (2008), Professionalität in der Schuldnerberatung. Handlungstypen im Vergleich. Wiesbaden: VS.

Weinkamm, P. (2004), EG-Beihilfen und die öffentlich-rechtlichen Kreditinstitute in Deutschland. Aachen: Shaker.

Wenzl, Chr. (2007), Das Örtlichkeitsprinzip im europäischen Binnenmarkt. Baden-Baden: Nomos.

Wissenschaftsförderung der Sparkassen-Finanzgruppe (Hrsg.) (2010), Sparkassenhistorisches Symposium 2008 Geschäftspolitische Steuerung. Die Sparkassen zwischen Renditeorientierung und Gemeinwohl. Stuttgart: Deutscher Sparkassen-Verlag.

Wysocki, J. (2005), Untersuchungen zur Wirtschafts- und Sozialgeschichte der deutschen Sparkassen im 19. Jahrhundert. Stuttgart: Deutscher Sparkassen Verlag. 